\title{
Parametrization of Neutrino Mixing Matrix in Tri-bimaximal Mixing Pattern
}

\author{
Nan Li \\ School of Physics, Peking University, Beijing 100871, China \\ Bo-Qiang $\mathrm{Ma*}$ \\ CCAST (World Laboratory), P.O. Box 8730, Beijing 100080, China \\ and School of Physics, Peking University, Beijing, 100871, China ${ }^{\dagger}$
}

\begin{abstract}
The neutrino mixing matrix is expanded in powers of a small parameter $\lambda$ in tri-bimaximal mixing pattern. We also present some applications of this parametrization, such as to the expression of the Jarlskog parameter $J$. Comparing with other parametrizations (such as the parametrization in bimaximal mixing pattern), this parametrization converges more quickly, but is of less symmetry.
\end{abstract}

PACS numbers: 14.60.Pq; 12.15.Ff; 14.60.Lm

In recent years, the mixing of different generations of neutrinos has been established by abundant experimental data. The KamLAND [1] and SNO 2] experiments showed that the long-existed solar neutrino deficit is due to the oscillation from $\nu_{e}$ to a mixture of $\nu_{\mu}$ and $\nu_{\tau}$ with a mixing angle approximately of $\theta_{\text {sol }} \approx 34^{\circ}$. The K2K [3] and Super-Kamiokande [4] experiments told us that the atmospheric neutrino anomaly is caused by the $\nu_{\mu}$ to $\nu_{\tau}$ oscillation with almost the largest mixing angle of $\theta_{\text {atm }} \approx 45^{\circ}$. On the other hand, the non-observation of the disappearance of $\bar{\nu}_{e}$ in the CHOOZ [5] experiment indicated that the mixing angle $\theta_{c h z}$ is smaller than $5^{\circ}$ at the best fit point [6, 7].

These experiments not only confirmed the oscillations of neutrinos, but also measured the mass-squared differences of the neutrino mass eigenstates [6] (the allowed ranges at $3 \sigma), 1.6 \times 10^{-3} \mathrm{eV}^{2}<\Delta m_{a t m}^{2}=\left|m_{3}^{2}-m_{2}^{2}\right|<$ $3.6 \times 10^{-3} \mathrm{eV}^{2}$, and $7.3 \times 10^{-5} \mathrm{eV}^{2}<\Delta m_{\text {sol }}^{2}=\mid m_{2}^{2}-$ $m_{1}^{2} \mid<9.3 \times 10^{-5} \mathrm{eV}^{2}$, where \pm correspond to the normal and inverted mass schemes respectively.

Just like the Cabibbo-Kobayashi-Maskawa (CKM) $\underline{9}$ matrix for quark mixing, the neutrino mixing matrix is described by the unitary Pontecorvo-Maki-NakawagaSakata (PMNS) 10] matrix $V$, which links the neutrino flavor eigenstates $\nu_{e}, \nu_{\mu}, \nu_{\tau}$ to the mass eigenstates $\nu_{1}$, $\nu_{2}, \nu_{3}$,

$$
\left(\begin{array}{c}
\nu_{e} \\
\nu_{\mu} \\
\nu_{\tau}
\end{array}\right)=\left(\begin{array}{ccc}
V_{e 1} & V_{e 2} & V_{e 3} \\
V_{\mu 1} & V_{\mu 2} & V_{\mu 3} \\
V_{\tau 1} & V_{\tau 2} & V_{\tau 3}
\end{array}\right)\left(\begin{array}{c}
\nu_{1} \\
\nu_{2} \\
\nu_{3}
\end{array}\right) .
$$

If neutrinos are of Dirac type, the neutrino mixing matrix can be written as follows (with three mixing angles and a Dirac CP-violating phase, analogous to that of quarks)

$V=\left(\begin{array}{ccc}c_{2} c_{3} & c_{2} s_{3} & s_{2} e^{-i \delta} \\ -c_{1} s_{3}-s_{1} s_{2} c_{3} e^{i \delta} & c_{1} c_{3}-s_{1} s_{2} s_{3} e^{i \delta} & s_{1} c_{2} \\ s_{1} s_{3}-c_{1} s_{2} c_{3} e^{i \delta} & -s_{1} c_{3}-c_{1} s_{2} s_{3} e^{i \delta} & c_{1} c_{2}\end{array}\right)$,

\footnotetext{
${ }^{\dagger}$ Mailing address

*Corresponding author; Electronic address: mabq@phy.pku.edu.cn
}

where $s_{i}=\sin \theta_{i}, c_{i}=\cos \theta_{i}$ (for $i=1,2,3$ ), and $\delta$ is the Dirac CP-violating phase. If neutrinos are of Majorana type, it is always feasible to parametrize the neutrino mixing matrix as a product of the Dirac neutrino mixing matrix and a diagonal phase matrix with two unremovable phase angles $\operatorname{diag}\left(e^{i \alpha}, e^{i \beta}, 1\right)$ [1] , where $\alpha, \beta$ are the Majorana $\mathrm{CP}$-violating phases. The Dirac CP-violating phase is associated with the neutrino oscillations, $\mathrm{CP}$ and $\mathrm{T}$ violation, and the Majorana $\mathrm{CP}$-violating phases are associated with the neutrinoless double beta decay, and lepton-number-violating processes 12 .

The three mixing angles $\theta_{\text {atm }}, \theta_{c h z}$, and $\theta_{\text {sol }}$ are related to $\theta_{1}, \theta_{2}$, and $\theta_{3}$, which describe the mixing between 2nd and 3rd, 3rd and 1st, 1st and 2nd generations of neutrinos. To a good degree of accuracy, $\theta_{a t m}=\theta_{1}$, $\theta_{c h z}=\theta_{2}$, and $\theta_{\text {sol }}=\theta_{3}$.

According to the results of the global analysis of the neutrino oscillation experimental data, the elements of the modulus of the neutrino mixing matrix are summarized as follows $[6]$

$$
|V|=\left(\begin{array}{ccc}
0.77-0.88 & 0.47-0.61 & <0.20 \\
0.19-0.52 & 0.42-0.73 & 0.58-0.82 \\
0.20-0.53 & 0.44-0.74 & 0.56-0.81
\end{array}\right)
$$

and the best fit points of the modulus of $V$ are [7]

$$
|V|=\left(\begin{array}{lll}
0.84 & 0.54 & 0.08 \\
0.44 & 0.56 & 0.72 \\
0.32 & 0.63 & 0.69
\end{array}\right)
$$

Quite different from quark mixing matrix, almost all the non-diagonal elements of the neutrino mixing matrix are large, only with the exception of $V_{e 3}$. So it is unpractical to expand the matrix in powers of one of the non-diagonal elements, like the Wolfenstein parametrization 13 of the quark mixing matrix. The quark mixing matrix is very near the unit matrix, but it is not a small modification to the unit matrix in neutrino mixing pattern. Several bases of neutrino mixing matrix are summarized as follows 14] (they all take some of the mixing 
angles as special values.)

$$
\begin{array}{r}
\left(\begin{array}{ccc}
\sqrt{2} / 2 & \sqrt{2} / 2 & 0 \\
-\sqrt{6} / 6 & \sqrt{6} / 6 & \sqrt{6} / 3 \\
\sqrt{3} / 3 & -\sqrt{3} / 3 & \sqrt{3} / 3
\end{array}\right),\left(\begin{array}{ccc}
\sqrt{3} / 2 & 1 / 2 & 0 \\
-\sqrt{2} / 4 & \sqrt{6} / 4 & \sqrt{2} / 2 \\
\sqrt{2} / 4 & -\sqrt{6} / 4 & \sqrt{2} / 2
\end{array}\right), \\
\left(\begin{array}{ccc}
\sqrt{2} / 2 & \sqrt{2} / 2 & 0 \\
-1 / 2 & 1 / 2 & \sqrt{2} / 2 \\
1 / 2 & -1 / 2 & \sqrt{2} / 2
\end{array}\right),\left(\begin{array}{ccc}
\sqrt{6} / 3 & \sqrt{3} / 3 & 0 \\
-\sqrt{6} / 6 & \sqrt{3} / 3 & \sqrt{2} / 2 \\
\sqrt{6} / 6 & -\sqrt{3} / 3 & \sqrt{2} / 2
\end{array}\right) .
\end{array}
$$

Therefore we may expand the neutrino mixing matrix around these bases. The third matrix is the bimaximal mixing pattern, and the expansions around it have been discussed by Rodejohann [15], Giunti and Tanimoto [16], and us [17.

The fourth matrix is the tri-bimaximal pattern. It was first conjectured by Wolfenstein [18] long ago, and was discussed by several other authors recently [19]. It is the best approximation to the neutrino mixing matrix, and its three mixing angles are $45^{\circ}, 0^{\circ}$ and $35.3^{\circ}$, which agree with the experimental data perfectly. So in this paper, we will expand the neutrino mixing matrix around it.

Comparing with Eq. (2), we can make an expansion of $V$ in powers of $\lambda$, which satisfies

$$
V_{e 2}=\sqrt{3} / 3-\lambda,
$$

where $\lambda$ measures the strength of the deviation of $V_{e 2}$ from the tri-bimaximal mixing pattern. Because the best fit point of $V_{e 2}$ is $0.53[\underline{6}], \lambda$ is a small parameter, which approximately equals to 0.05 , and this expansion is reasonable and will converge quickly.

For the range of $\lambda$, from the analyses of the experimental data [6], we have $0.51<V_{e 2}<0.55$ (the allowed range at $1 \sigma)$. So $0.51<\sqrt{3} / 3-\lambda<0.55$, and we can get $0.03<\lambda<0.07$. Similarly, $-0.03<\lambda<0.1$ (the allowed range at $3 \sigma)$.

Similarly, with the global analyses on the experimental data, the best fit point of $\left|V_{\mu 3}\right|^{2}$ is 0.52 [7]. Therefore we have $V_{\mu 3}=0.72$, and the deviation of $V_{\mu 3}$ from $\sqrt{2} / 2$ is very small, so we can set

$$
V_{\mu 3}=\sqrt{2} / 2+a \lambda .
$$

Thus $a \lambda \sim 0.013$, and $a \sim 0.3$.

Furthermore, since $\theta_{2}$ is rather small (with the global analyses, $\left|V_{e 3}\right|<0.25$ (the allowed range at $3 \sigma$ ), and with the best fit point $\left|V_{e 3}\right|=0.08$ []],[]$\left.]\right)$, we can set

$$
V_{e 3}=b \lambda e^{i \delta} \text {. }
$$

So $b \sim 1.5$. Due to the uncertainty of the value of $\theta_{2}$, only the upper bound 0.25 is meaningful in phenomenological anlyses, and the parametrization in Eq. (5) is only an assumption, however, we can adjust the value of $b$ to satisfy the best fit point of $V_{e 3}$, which can be determined by the long baseline experiments [20] in the future.

Altogether, there are four parameters here, $a, b, \lambda$ and $\delta$. They can describe the neutrino mixing matrix completely, both the real and the imaginary parts.

Now we will calculate all the $s_{i}$ and $c_{i}$ (for $i=1,2,3$ ) to the order of $\lambda^{3}$. From Eq. (5), $s_{2}=b \lambda$, we have

$$
c_{2}=\sqrt{1-s_{2}^{2}}=1-\frac{1}{2} b^{2} \lambda^{2} .
$$

From Eq. (4), we have

$$
s_{1} c_{2}=V_{\mu 3}=\sqrt{2} / 2+a \lambda,
$$

using Eq. (6), we get

$$
s_{1}=\frac{\sqrt{2}}{2}+a \lambda+\frac{\sqrt{2}}{4} b^{2} \lambda^{2}+\frac{1}{2} a b^{2} \lambda^{3} .
$$

Similarly,

$$
\begin{aligned}
c_{1}= & \frac{\sqrt{2}}{2}-a \lambda-\left(\sqrt{2} a^{2}+\frac{\sqrt{2}}{4} b^{2}\right) \lambda^{2}-\left(2 a^{3}+\frac{3}{2} a b^{2}\right) \lambda^{3} \\
s_{3}= & \frac{\sqrt{3}}{3}-\lambda+\frac{\sqrt{3}}{6} b^{2} \lambda^{2}-\frac{1}{2} b^{2} \lambda^{3}, \\
c_{3}= & \frac{\sqrt{6}}{3}+\frac{\sqrt{2}}{2} \lambda-\left(\frac{3 \sqrt{6}}{8}+\frac{\sqrt{6}}{12} b^{2}\right) \lambda^{2} \\
& +\left(\frac{9 \sqrt{2}}{16}+\frac{5 \sqrt{2}}{8} b^{2}\right) \lambda^{3} .
\end{aligned}
$$

Thus we obtain all the trigonometric functions of the three mixing angles.

So we can get all the elements of the neutrino mixing matrix straightforwardly (to the order of $\lambda^{2}$ ),

$$
\begin{aligned}
V_{e 1}= & \frac{\sqrt{6}}{3}+\frac{\sqrt{2}}{2} \lambda-\left(\frac{3 \sqrt{6}}{8}+\frac{\sqrt{6} b^{2}}{4}\right) \lambda^{2}, \\
V_{e 2}= & \frac{\sqrt{3}}{3}-\lambda \\
V_{e 3}= & b \lambda e^{i \delta} \\
V_{\mu 1}= & -\frac{\sqrt{6}}{6}+\left(\frac{\sqrt{2}}{2}+\frac{\sqrt{3} a}{3}\right) \lambda-\left(a-\frac{\sqrt{6} a^{2}}{3}\right) \lambda^{2} \\
& -\left[\frac{\sqrt{3}}{3} b \lambda+\left(\frac{b}{2}+\frac{\sqrt{6} a b}{3}\right) \lambda^{2}\right] e^{i \delta}, \\
V_{\mu 2}= & \frac{\sqrt{3}}{3}+\left(\frac{1}{2}-\frac{\sqrt{6} a}{3}\right) \lambda-\left(\frac{3 \sqrt{3}}{8}+\frac{\sqrt{2} a}{2}+\frac{2 \sqrt{3} a^{2}}{3}\right. \\
& \left.+\frac{\sqrt{3} b^{2}}{4}\right) \lambda^{2}-\left[\frac{\sqrt{6}}{6} b \lambda-\left(\frac{\sqrt{2} b}{2}-\frac{\sqrt{3} a b}{3}\right) \lambda^{2}\right] e^{i \delta}, \\
V_{\mu 3}= & \frac{\sqrt{2}}{2}+a \lambda, \\
V_{\tau 1}= & \frac{\sqrt{6}}{6}-\left(\frac{\sqrt{2}}{2}-\frac{\sqrt{3} a}{3}\right) \lambda-\left(a-\frac{\sqrt{6} b^{2}}{6}\right) \lambda^{2} \\
& -\left[\frac{\sqrt{3}}{3} b \lambda+\left(\frac{b}{2}-\frac{\sqrt{6} a b}{3}\right) \lambda^{2}\right] e^{i \delta}, \\
V_{\tau 2}= & -\frac{\sqrt{3}}{3}-\left(\frac{1}{2}+\frac{\sqrt{6} a}{3}\right) \lambda+\left(\frac{3 \sqrt{3}}{8}-\frac{\sqrt{2} a}{2}-\frac{\sqrt{3} b^{2}}{12}\right) \lambda^{2} \\
& -\left[\frac{\sqrt{6}}{6} b \lambda-\left(\frac{\sqrt{2} b}{2}+\frac{\sqrt{3} a b}{3}\right) \lambda^{2}\right] e^{i \delta}, \\
V_{\tau 3}= & \frac{\sqrt{2}}{2}-a \lambda-\left(\sqrt{2} a^{2}+\frac{\sqrt{2}}{2} b^{2}\right) \lambda^{2} . \\
&
\end{aligned}
$$

Then we can expand the neutrino mixing matrix in powers of $\lambda$ (to the order of $\lambda^{2}$ ), 


$$
\begin{aligned}
V= & \left(\begin{array}{ccc}
\frac{\sqrt{6}}{3} & \frac{\sqrt{3}}{3} & 0 \\
-\frac{\sqrt{6}}{6} & \frac{\sqrt{3}}{3} & \frac{\sqrt{2}}{2} \\
\frac{\sqrt{6}}{6} & -\frac{\sqrt{3}}{3} & \frac{\sqrt{2}}{2}
\end{array}\right)+\lambda\left(\begin{array}{ccc}
\frac{\sqrt{2}}{2} & -1 & b e^{i \delta} \\
\left(\frac{\sqrt{2}}{2}+\frac{\sqrt{3} a}{3}\right)-\frac{\sqrt{3}}{3} b e^{i \delta} & \left(\frac{1}{2}-\frac{\sqrt{6} a}{3}\right)-\frac{\sqrt{6}}{6} b e^{i \delta} & a \\
-\left(\frac{\sqrt{2}}{2}-\frac{\sqrt{3} a}{3}\right)-\frac{\sqrt{3}}{3} b e^{i \delta} & -\left(\frac{1}{2}+\frac{\sqrt{6} a}{3}\right)-\frac{\sqrt{6}}{6} b e^{i \delta} & -a
\end{array}\right) \\
& +\lambda^{2}\left(\begin{array}{cccc}
-\left(a-\frac{\sqrt{6} a^{2}}{3}\right)-\left(\frac{\sqrt{6}}{2}+\frac{\sqrt{6} a b}{3}\right) e^{i \delta} & -\left(\frac{3 \sqrt{3}}{8}+\frac{\sqrt{2} a}{2}+\frac{2 \sqrt{3} a^{2}}{3}+\frac{\sqrt{3} b^{2}}{4}\right)+\left(\frac{\sqrt{2} b}{2}-\frac{\sqrt{3} a b}{3}\right) e^{i \delta} & 0 \\
-\left(a-\frac{\sqrt{6} b^{2}}{6}\right)-\left(\frac{b}{2}-\frac{\sqrt{6} a b}{3}\right) e^{i \delta} & \left(\frac{3 \sqrt{3}}{8}-\frac{\sqrt{2} a}{2}-\frac{\sqrt{3} b^{2}}{12}\right)+\left(\frac{\sqrt{2} b}{2}+\frac{\sqrt{3} a b}{3}\right) e^{i \delta} & -\left(\sqrt{2} a^{2}+\frac{\sqrt{2}}{2} b^{2}\right)
\end{array}\right) \\
& +\cdots
\end{aligned}
$$

Now we will see the meaning of every order in the expansion of $V$.

1. The term of $\lambda^{0}$ is the approximation of the lowest order, where the mixing angles are of $45^{\circ}, 0^{\circ}$ and $35.3^{\circ}$. We call this the tri-bimaximal mixing pattern, and it is nearest to the experimental data among the bases with special mixing angles.

2. The term of $\lambda^{1}$ indicates the deviation of the neutrino mixing matrix from the tri-bimaximal mixing pattern. Also it shows the effect of $\mathrm{CP}$ violation. Because $\mathrm{CP}$ violation is described by the element $V_{e 3}$ 21], and in the terms of $\lambda^{0}, V_{e 3}=0$, the degree of $\mathrm{CP}$ violation is of the order $\lambda^{1}$ in our parametrization.

3. The term of $\lambda^{2}$ and so on are the modifications of higher orders.

In this parametrization, several other corresponding observable quantities associated with the elements of the neutrino mixing matrix can be expressed in relatively simple forms.

1. The Jarlskog parameter $J[22] . J$ is the rephasinginvariant measurement of the lepton $\mathrm{CP}$ violation. The Majorana CP-violating phases can be removed away by redefining the phases of the Dirac fields, so only $\delta$ is associated with CP violation. $J=\operatorname{Im}\left(V_{e 2} V_{\mu 3} V_{e 3}^{*} V_{\mu 2}^{*}\right)=$ $s_{1} s_{2} s_{3} c_{1} c_{2}^{2} c_{3} \sin \delta$. In our parametrization, $J$ can be expressed in a simple form (to the order of $\lambda^{2}$ ),

$$
J=\frac{\sqrt{2}}{6} b \lambda \sin \delta\left(1-\frac{\sqrt{3}}{2} \lambda\right) .
$$

Because $s_{1}, c_{1}, s_{3}, c_{3}$ have the factors $\frac{\sqrt{2}}{2}, \frac{\sqrt{2}}{2}, \frac{\sqrt{3}}{3}, \frac{\sqrt{6}}{3}$, there are four factors smaller than 1 in $J$. So the degree of the lepton CP violation is suppressed four times, $\left(\frac{\sqrt{2}}{2}\right)^{2} \frac{\sqrt{3}}{3} \frac{\sqrt{6}}{3}=\frac{\sqrt{2}}{5}$. Again, $J$ is suppressed by the factor $b \lambda \sim 0.08$ [7]. We can determine the range of $J$, $J \sim 0.018$. (here we take $\lambda \sim 0.05$ and $\sin \delta \sim 1$.)

2. The effective Majorana mass term $\langle m\rangle_{e e}$. In the neutrinoless double beta decay, the effective Majorana mass term is defined as follows

$$
\langle m\rangle_{e e} \equiv\left|m_{1} V_{e 1}^{2} e^{2 i \alpha}+m_{2} V_{e 2}^{2} e^{2 i \beta}+m_{3} V_{e 3}^{2}\right| .
$$

Using Eq. (9), we get

$$
\begin{aligned}
\langle m\rangle_{e e}= & \mid \frac{1}{3}\left(2 m_{1} e^{2 i \alpha}+m_{2} e^{2 i \beta}\right) \\
& +\frac{2 \sqrt{3}}{3} \lambda\left(m_{1} e^{2 i \alpha}-m_{2} e^{2 i \beta}\right) \\
& -\lambda^{2}\left[\left(m_{1} e^{2 i \alpha}-m_{2} e^{2 i \beta}\right)+b^{2}\left(m_{1} e^{2 i \alpha}-m_{3} e^{2 i \delta}\right)\right] \mid .
\end{aligned}
$$

We can see that the coefficients of the three terms show the influences of the three orders of $\lambda$. Only $m_{1}$ and $m_{2}$ are important to the value of $\langle m\rangle_{e e}$, and the influence of $m_{3}$ almost vanish if the masses of the three mass eigenstates are nearly degenerated, because the coefficient of it $b^{2} \lambda^{2}$ is of $10^{-3}$.

3 . The effective mass terms of neutrinos. The effective mass terms of neutrinos can be defined as follows (here we take electron neutrino for example.)

$$
\langle m\rangle_{e}^{2} \equiv m_{1}^{2}\left|V_{e 1}\right|^{2}+m_{2}^{2}\left|V_{e 2}\right|^{2}+m_{3}^{2}\left|V_{e 3}\right|^{2} .
$$

Using Eq. (9), we get

$$
\begin{aligned}
\langle m\rangle_{e}^{2}= & \frac{1}{3}\left(2 m_{1}^{2}+m_{2}^{2}\right)-\frac{2 \sqrt{3}}{3} \lambda\left(m_{2}^{2}-m_{1}^{2}\right) \\
& +\lambda^{2}\left[\left(m_{2}^{2}-m_{1}^{2}\right)+b^{2}\left(m_{3}^{2}-m_{1}^{2}\right)\right] .
\end{aligned}
$$

Again, the coefficients of the three terms show the influences of the three orders of $\lambda$. Noting that $\Delta m_{\text {sol }}^{2}=$ $\left|m_{2}^{2}-m_{1}^{2}\right|$ and $\Delta m_{a t m}^{2}=\left|m_{3}^{2}-m_{2}^{2}\right|$, we can rewrite Eq. (11) into

$$
\begin{aligned}
\langle m\rangle_{e}^{2}= & m_{1}^{2}+\left[\frac{1}{3}-\frac{2 \sqrt{3}}{3} \lambda+\left(b^{2}+1\right) \lambda^{2}\right]\left(m_{2}^{2}-m_{1}^{2}\right) \\
& +b^{2} \lambda^{2}\left(m_{3}^{2}-m_{2}^{2}\right) \\
= & m_{1}^{2} \pm\left[\frac{1}{3}-\frac{2 \sqrt{3}}{3} \lambda+\left(b^{2}+1\right) \lambda^{2}\right] \Delta m_{\text {sol }}^{2} \\
& \pm b^{2} \lambda^{2} \Delta m_{\text {atm }}^{2}
\end{aligned}
$$

where the first sign of " \pm " should be chosen as "+" if we accept $m_{2}>m_{1}$ because of Mikheyev-SmirnovWolfenstein (MSW) 23] matter effect on solar neutrinos. We can see from Eq. (12) that $\langle m\rangle_{e}^{2}$ is directly related with the masses and the mass-squared differences of neutrinos. So these two kinds of different observable quantities are associated together in our parametrization. 
If we can separately measure $\Delta m_{a t m}^{2}, \Delta m_{\text {sol }}^{2}$, and $\langle m\rangle_{e}^{2}$ to a good degree of accuracy, we can fix the value of $m_{1}$, which will help us determine the absolute mass of neutrino ultimately.

Finally, we will give some discussion and comparison between the different methods in parametrizing the neutrino mixing matrix.

For the quark mixing, all the non-diagonal elements are small, so it is practical to expand the quark mixing matrix around the unit matrix. But the case is clearly different for the neutrino mixing. So if we still present Wolfenstein-like parametrization for the neutrino mixing matrix (as Xing did [24]), we have to use much higher orders of the non-diagonal elements. Hence it is necessary for us to find new bases for the expansion of the neutrino mixing matrix.

Among all the matrices with special mixing angles, the tri-biamaximal mixing pattern seems to be the best one. So it is natural to expand the neutrino mixing matrix around it. This is the main point of our paper. But just due to the smallness of $\lambda(\lambda \sim 0.05)$, the expansion converges so quickly that the modulus of the matrix is rather small only to the order of $\lambda^{2}$. However, in the bimaximal case, we can expand the neutrino mixing matrix to higher orders, and can see different physical effects in different orders, because $\lambda$ there is larger $(\lambda \sim 0.1)[17$. Moreover, the expansion in tri-bimaximal mixing pattern has less symmetry than the expansion in bimaximal mix- ing pattern, because the mixing angles are not the same here.

Altogether, we can see that there are advantages and deficiencies at the same time in both the expansions in tri-bimaximal and bimaxiamal mixing patterns, and the adoption of which of them should be determined by more and more precise experimental data.

In summary, although all sorts of parametrization of the neutrino mixing matrix are not based on any deep theoretical foundation and are equivalent mathematically, and applying any of them is arbitrary, however, it is quite likely that some particular parametrization is useful in making sense of experimental data. Furthermore, we can express some other observable quantities in a relatively simple form, and can link several different kinds of observable quantities together. This is the purpose of our parametrization.

\section{Acknowledgments}

We are very grateful to Prof. Xiao-Gang He for his stimulating suggestions and discussions. This work is partially supported by National Natural Science Foundation of China under Grant Numbers 10025523 and 90103007.
[1] KamLAND Collaboration, K. Eguchi et al., Phys. Rev. Lett. 90, 021802 (2003).

[2] SNO Collaboration, S.N. Ahmed et al., Phys. Rev. Lett. 92, 181301 (2004).

[3] K2K Collaboration, M.H. Ahn et al., Phys. Rev. Lett. 90, 041801 (2003).

[4] C.K. Jung, C. McGrew, T. Kajita, and T. Mann, Anna. Rev. Nucl. Part. Sci. 51, 451 (2001); Super-Kamiokande Collaboration, Y. Hayato, talk at HEP2003 International Europhysics Conference, Aachen, Germany, 2003, http://eps2003.physik.rwth-aachen.de

[5] CHOOZ Collaboration, M. Apollonio et al., Phys. Lett. B 420, 397 (1998); Palo Verde Collaboration, F. Boehm et al., Phys. Rev. Lett. 84, 3764 (2000).

[6] M.C. Gonzalez-Garcia, hep-ph/0410030

[7] G. Altarelli, hep-ph/0410101

[8] G. Altarelli and F. Feruglio, hep-ph/0405048 New J. Phys. 6, 106 (2004).

[9] N. Cabibbo, Phys. Rev. Lett. 10, 531 (1963); M. Kobayashi and T. Maskawa, Prog. Theor. Phys. 149, 652 (1973).

[10] B. Pontecorvo, Sov. Phys. JETP 7, 172 (1958); Z. Maki, M. Nakawaga, and S. Sakata, Prog. Theor. Phys. 28, 870 (1962).

[11] J. Schechter and J.W.F. Valle, Phys. Rev. D 22, 2227 (1980); S.M. Bilenky, J. Hosek, and S.T. Petsov, Phys. Lett. B 94, 495 (1980).

[12] J. Schechter and J.W.F. Valle, Phys. Rev. D 23, 1666
(1981); Phys. Rev. D 24, 1883 (1981); Phys. Rev. D 25, 283 (1982).

[13] L. Wolfenstein, Phys. Rev. Lett. 51, 1945 (1983).

[14] Z.Z. Xing, Int. J. Mod. Phys. A 19, 1 (2004).

[15] W. Rodejohann, Phys. Rev. D 69, 033005 (2004); Nucl. Phys. B 687, 31 (2004).

[16] C. Giunti and M. Tanimoto, Phys. Rev. D 66, 053013 (2002); Phys. Rev. D 66, 113006 (2002).

[17] N. Li and B.-Q. Ma, Phys. Lett. B 600, 248 (2004).

[18] L. Wolfenstein, Phys. Rev. D 18, 958 (1978).

[19] P.F. Harrison, D.H. Perkins, and W.G. Scott, Phys. Lett. B 530, 167 (2002); Z.Z. Xing, Phys. Lett. B 533, 85 (2002); X.G. He and A. Zee, Phys. Lett. B 560, 87 (2003); C.I. Low and R.R. Volkas, Phys. Rev. D 68, 033007 (2003); A. Zee, Phys. Rev. D 68, 093002 (2003).

[20] H. Minakata,H. Sugiyama, O. Yasuda, K. Inoue, and F. Suekane, Phys. Rev. D 68033017 (2003); P. Huber et al., Nucl. Phys. B 665487 (2003).

[21] F.J. Gilman, K. Kleinknecht, and B. Renk, Phys. Rev. D 66, 010001 (2002); L. Wolfenstein, Phys. Rev. D 66, 010001 (2002).

[22] C. Jarlskog, Phys. Rev. Lett. 55, 1039 (1985); Z. Phys. C 29, 491 (1985).

[23] S.P. Mikheyev and A.Yu. Smirnov, Sov. J. Nucl. Phys. 42913 (1985); L. Wolfenstein, Phys. Rev. D 172369 (1978).

[24] Z.Z. Xing, J. Phys. G 29, 2227 (2003). 